\title{
Tratamento de Estenose Subaórtica em Membrana por Cateter Balão
}

\author{
José Luiz B. Jacob, N ilton Carlos S. Machado, W ilson Miguel C. Coelho, Sérgio Aloisio C. Garzon
}

São José do Rio Preto, SP

\begin{abstract}
Objetivo - Avaliar os resultados imediatos e a médio prazo do tratamento da estenose subaórtica em membrana através da dilatação percutânea por cateter balão.

Métodos - Os 14 pacientes, com idade média de 11,4+5,2 anos, foram selecionados pelo estudo ecodopplercardiográfico, mediante evidência de membrana subaórtica de fina espessura e distante das válvulas aórticas, ausência de componente muscular associado ou insuficiência aórtica (IAo) importante. Após a medida do gradiente e comprovação dos achados pela cineangiocardiografia, as dilatações eram feitas por insuflação manual e rápida até o desaparecimento da constricção do balão. O diâmetro do balão era no máximo igual ao da via de saída de ventrículo esquerdo, medida logo abaixo da valva aórtica. Manometria, ventriculografia esquerda e aortograma eram repetidos. Ecodopplercardiograma era realizado no dia seguinte, após 3 meses e a cada 6 meses após o procedimento.

Resultados - Os 17 procedimentos foram realizados com sucesso. O gradiente médio da amostra foi $76,1 \pm$ 21,2mmHg (41-115) pré dilatação e 29,8 $\pm 8,8 \mathrm{mmHg}$ (13$45)$ pós dilatação $(p<0,01)$. Não houve aumento da IAo pós procedimento. Doze pacientes receberam alta em $24 \mathrm{~h}$ e 2 apresentaram oclusão de artéria femoral, tratados cirurgicamente. Não houve óbito imediato ou tardio. No acompanhamento de 33,3+23,6 meses (1-75) ocorreu reestenose em quatro pacientes, sendo três deles redilatados com sucesso.

Conclusão - Em casos selecionados, o procedimento é seguro e eficaz e a ocorrência de reestenose pode ser tratada com nova dilatação percutânea.
\end{abstract}

Palavras-chaves: tratamento percutâneo, dilatação percutânea, estenose subaórtica fixa

\section{Balloon Dilatation for the Treatment of Membranous Subaortic Stenosis}

Purpose - This study sought to evaluate the immediate and follow-up results of percutaneous balloon dilatation for the treatment of membranous subaortic stenoses.

Methods - Fourteen patients with mean age $11.4 \pm 5.2$ years, were submitted to the procedure. They were selected when the echocardiogram showed a thin subaortic membrane that was far from the aortic valve, no fibro-muscular obstruction and only mild or moderate aortic regurgitation. After measuring the pressure gradient and analysis of the angiographic features, the balloon dilatation was made by applying a fast manual inflation until the balloon waist disappeared. The balloon diameter was the same as that of the outflow tract of left ventricle, immediately bellow the aortic valve. Pressure measurement, left ventriculogram and aortogram were repeated. Doppler echocardiogram was repeated in the following day, after 3 months and every 6 months thereafter.

Results - All 17 procedures were successful. The mean gradient was $76.1 \pm 21.2 \mathrm{mmHg}$ before and $29.8 \pm 8.8 \mathrm{mmHg}$ after dilatation $(p<0.01)$. There was no increase in aortic regurgitation or death after the procedure or during the follow-up. Twelve patients were discharged $24 \mathrm{~h}$ after the procedure. Surgical treatment for femoral artery thrombosis was performed in 2 patients. In the follow-up of $33.3 \pm 23.6$ months, 4 patients developed restenosis and 3 of them were submitted to successful redilatation.

Conclusion - We conclude that in selected cases, the procedure is safe and effective, and restenosis may be treated by percutaneous balloon redilatation.

Key-words: percutaneous treatment, percutaneous dilatation, discrete subaortic stenosis

Arq Bras Cardiol, volume 70 (n' 1), 25-28, 1998

Instituto de Moléstias Cardiovasculares de São José do Rio Preto Correspondência: José Luiz B. Jacob - Rua Castelo D'Água, 3030 - 15015-210 São José do Rio Preto, SP

Recebido para publicação em 6/07/97

Aceito em 31/10/97
A estenose subaórtica em membrana tem evolução progressiva com aumento do gradiente na via de saída do ventrículo esquerdo(VE)e, também, da insuficiência valvar aórtica ${ }^{1,2}$. Este potencial progressivo se deve à possibilidade de extensão muscular da lesão, intensificando e tornan- 
do mais complexo o processo obstrutivo, e ao agravamento de regurgitação aórtica, decorrente da lesão de jato produzida pelo fluxo sanguíneo excêntrico, através do orifício estenótico $^{3-5}$.

Dilatação por cateter balão para tratamento desta doença foi introduzida como opção ao tratamento cirúrgico e neste artigo apresentamos nossa experiência com este procedimento.

\section{Métodos}

Entre agosto/90 e janeiro/97, foram realizados 17 procedimentos de dilatação de estenose subaórtica em membrana em 14 pacientes com idade média de 11,4+5,2 anos (422), sendo 8 do sexo feminino e todos selecionados, a partir do estudo ecodopplercardiográfico, mediante evidência de imagem de membrana subaórtica de fina espessura, distante das válvulas aórticas, ausência de componente muscular associado ou insuficiência aórtica (IAo) importante. Três pacientes haviam sido submetidos a procedimentos anteriores: um fechamento de comunicação interventricular e ressecção de membrana subaórtica, uma correção términoterminal de coarctação de aorta e uma valvoplastia pulmonar por cateter balão.

Sedação com ketamina e fentanil foi usada em 13 pacientes, sendo dispensada em apenas um caso. Através de dissecção de artéria femoral, um cateter com furo terminal era introduzido para tomada de pressões no VE e aorta, obtendo-se assim o gradiente entre a via de entrada e a via de saída do VE. Em seguida, o cateter era retirado e um cateter angiográfico NIH ou pig-tail (USCI) era introduzido para realização de cineangioventriculografia esquerda (fig. 1) e angiografia de raiz de aorta. Após confirmação dos achados ecocardiográficos, um cateter com furo terminal era posicionado no VEe um guia $0,035 \mathrm{de} 260 \mathrm{~cm}$ de comprimento (Cook) era introduzido através dele.

Obtida uma posição adequada do guia, o cateter era retirado e um cateter balão de diâmetro igual ou $1 \mathrm{~mm}$ menor que o da via de saída de VE (medida imediatamente abaixo da valva aórtica) era avançado sobre o fio guia e posicio-

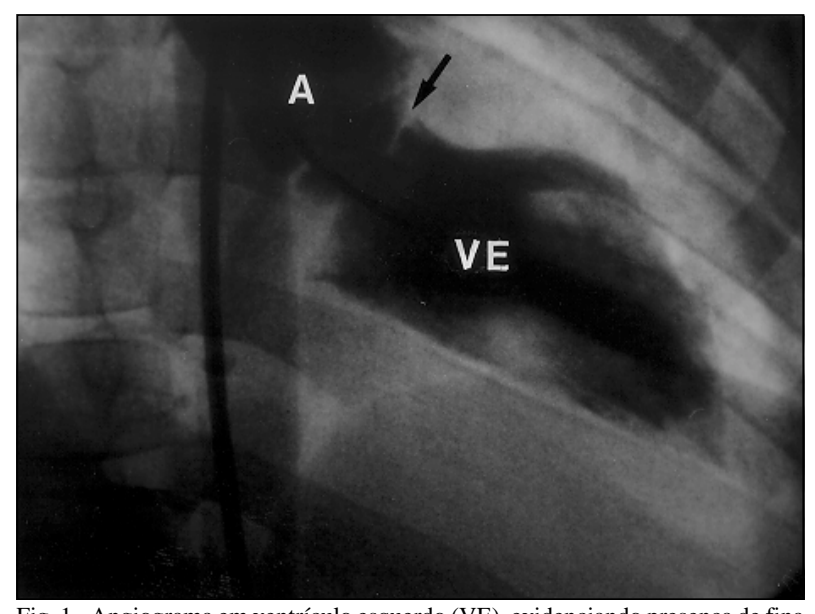

Fig. 1 - Angiograma em ventrículo esquerdo (VE), evidenciando presença de fin membrana subaórtica (seta) e opacificação de aorta (Ao).

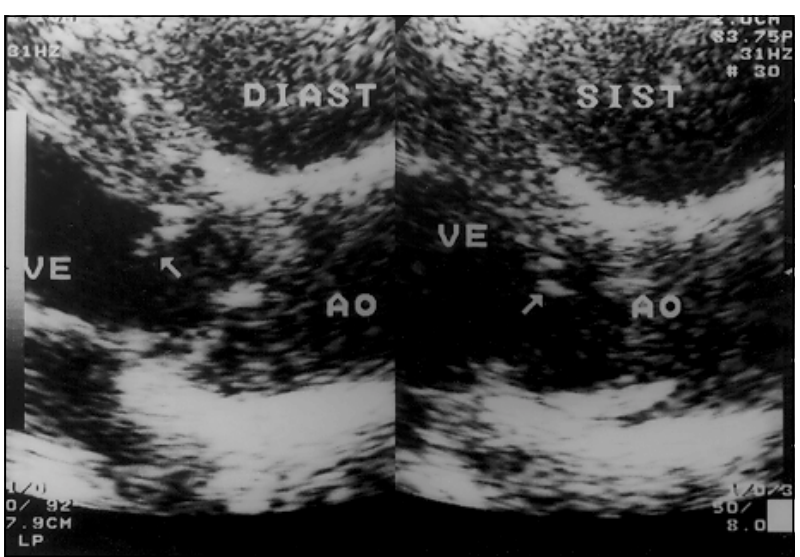

Fig. 2 -Ecocardiograma pós dilatação, evidenciando aspecto de movimentação ampla da membrana subaórtica (seta) em diástole para o ventrículo esquerdo (VE) e em sístole em direção de Aorta (Ao).

nado para insuflação. Insuflações eram feitas manualmente e repetidas até que desaparecesse a constricção do balão durante a insuflação, o que em todos os casos acompanhava-se de livre movimentação do balão através da via de saída do VE e da valva aórtica. Todos os procedimentos foram realizados com cateter balão único.

Após a retirada do cateter balão, sobre o guia mantido no VE um cateter de furo terminal era posicionado na cavidade ventricular. Após retirada do guia, uma nova manometria era realizada para avaliação do gradiente residual.

Novamente o cateter era trocado por um cateter angiográfico para realização de nova ventriculografia esquerda e aortograma.

Ecodopplercardiograma era realizado no dia seguinte, após três meses e a cada seis meses após o procedimento.

Foi investigada a mortalidade, bem como complicações (endocardite infecciosa (EI), reestenose e progressão de IAo) no período de seguimento de 33,3 $\pm 23,6$ meses (175). Profilaxia de EI era fortemente estimulada para os pacientes, seguindo os critérios da American Heart Association. Consideramos como reestenose, os casos onde houve aumento do gradiente em mais de $50 \%$ do obtído pós dilatação ou quando gradientes abaixo de $30 \mathrm{mmHg}$ após o procedimento, atingiram $45 \mathrm{mmHg}$ ou mais no acompanhamento.

\section{Resultados}

Os 17 procedimentos foram realizados com sucesso, obtendo-se redução de $50 \%$ ou mais no gradiente pressórico. O gradiente médio da amostra era 76,1 $21,2 \mathrm{mmHg}$ (41-115) pré-dilataçãoe $29,8 \pm 8,8 \mathrm{mmHg}(13-45)$ pós-dilatação $(\mathrm{p}<0,01)$.

Reestenose ocorreu em quatro $(28,5 \%)$ pacientes num intervalo de tempo de 12, 40, 65 e 72 meses, respectivamente, e apenas um deles foi encaminhado à cirurgia por opção familiar. Três foram submetidos à redilatação com sucesso, sempre se obtendo redução do gradiente de $50 \%$ ou mais em relação ao anterior à primeira dilatação.

A idade dos pacientes, o gradiente pré e pós procedi- 


\begin{tabular}{|c|c|c|c|c|c|}
\hline $\begin{array}{c}\text { Idade } \\
\text { (anos) }\end{array}$ & Sexo & $\Delta$ pré & $\Delta$ pós & $\begin{array}{l}\text { I Ao } \\
\text { pré }\end{array}$ & $\begin{array}{l}\text { I Ao } \\
\text { pós }\end{array}$ \\
\hline 10 & f & 83 & 40 & + & + \\
\hline 4 & $\mathrm{f}$ & 41 & 17 & + & + \\
\hline 9 & $\mathrm{f}$ & 74 & 29 & + & + \\
\hline 22 & $\mathrm{~m}$ & 96 & 45 & + & + \\
\hline 12 & $\mathrm{~m}$ & 115 & 35 & + & + \\
\hline 17 & $\mathrm{~m}$ & 115 & 20 & + & + \\
\hline $23 *$ & $\mathrm{~m}$ & 77 & 45 & + & + \\
\hline 8 & $\mathrm{~m}$ & 45 & 22 & + & + \\
\hline 13 & $\mathrm{f}$ & 60 & 35 & + & + \\
\hline 7 & $\mathrm{~m}$ & 56 & 27 & + & + \\
\hline 6 & $\mathrm{~m}$ & 55 & 27 & + & + \\
\hline 11 & $\mathrm{f}$ & 75 & 30 & + & + \\
\hline 7 & $\mathrm{f}$ & 99 & 20 & + & + \\
\hline 7 & f & 83 & 29 & + & + \\
\hline $16 *$ & $\mathrm{f}$ & 83 & 42 & + & + \\
\hline 10 & $\mathrm{~m}$ & 78 & 24 & + & + \\
\hline $12 *$ & $\mathrm{~m}$ & 60 & 20 & + & + \\
\hline $\begin{array}{l}\Delta \text { - gradi } \\
\text { redilataç }\end{array}$ & Ao- & ência & $\mathrm{a} ;+\mathrm{c}$ & ; ++ & ada; * \\
\hline
\end{tabular}

mento e o grau de IAo pré e pós-dilatação, incluindo-se as redilatações, são apresentados na tabela I, notando-se que o procedimento não causou aumento da IAo em nenhum caso, havendo inclusive diminuição em um paciente.

O ecocardiograma mostrou aspecto de vibração da membrana por rotura, após 14 procedimentos (fig. 2).

Não ocorreu óbito durante os procedimentos e 12 pacientes tiveram alta $24 \mathrm{~h}$ após a dilatação. Dois pacientes apresentaram oclusão de artéria femoral, sendo submetidos à trombectomia e restauração arterial com sucesso. Não houve óbito tardio. O único paciente que abandonou o acompanhamento ecocardiográfico há 24 meses, sabe-se estar, por informações telefônicas, com atividade normal.

No acompanhamento de $13(92,8 \%)$ pacientes por um período de $33,3 \pm 23,6$ meses (1-75), o seguimento ecodopplercardiográfico mostrou gradiente médio da amostra de 36,0 $\pm 21,5 \mathrm{mmHg}$ (11-83), incluindo-se os casos de reestenose, que é significativamente inferior à pré-dilatação ( $\mathrm{p}<0,02)$ e ausência de progressão da IAo. Nenhum paciente desenvolveu EI durante o seguimento.

\section{Discussão}

Embora o tratamento cirúrgico da estenose subaórtica em membrana seja bem estabelecido para alívio da obstrução, recidiva com progressivo aumento do gradiente na via de saída do ventrículo tem sido encontrada com freqüência ${ }^{2,5-7}$. Apesar dos avanços técnicos, a mortalidade da correção cirúrgica é pouco menor que $5 \%$ e gradientes residuais podem obrigar a reoperação precoce ${ }^{1,2}$. Diante das dúvidas quanto a evolução pós-cirúrgica e em relação ao momento ideal para se indicar a cirurgia ${ }^{2,7,8}$, Suarez de Lezo e col $^{4} \mathrm{e}$ Lababid e col ${ }^{5}$, relataram as primeiras séries de pacientes submetidos à dilatação por cateter balão, mostrando resultados satisfatórios nas obstruções em membrana. A partir de então, a utilização de dilatação percutânea tem sido ampliada ${ }^{1,3,4,9}$ e, no Brasil, Jacob e col ${ }^{10,11}$ foram pioneiros no uso desta técnica com resultados iniciais animadores. Em nossa experiência, a associação de dados ecocardiográficos e angiográficos tem permitido uma seleção adequada dos pacientes. $\mathrm{O}$ valor da ecocardiografia no diagnóstico desta doença e na caracterização de seus aspectos morfológicos é bem definido, bem como seu papel no seguimento pós intervenção percutânea ou cirúrgica ${ }^{3,12}$. Os casos ideais, segundo avaliações ecodopplercardiográfica e angiográfica devem ter uma membrana de espessura fina $(<3 \mathrm{~mm})$ e distante da valva aórtica, ausência de componente muscular envolvido no processo obstrutivo e IAo, no máximo, moderada.

O alívio do gradiente pressórico é obtido por rotura de membrana que, em nossa opinião, é muito melhor definida pelo aspecto vibrátil ao ecodopplercardiograma que a mesma adquire após a dilatação.

Raramente encontramos imagens angiográficas de vibração à rotura da membrana relatadas na literatura ${ }^{3}$.

Nosso índice de reestenose de $28,5 \%$, não difere do encontrado na literatura, seja através do tratamento cirúrgico ou da dilatação percutânea ${ }^{3,6,7}$, tendo sido mais precoce (12 meses) em um paciente onde o ecocardiograma evidenciava redução do gradiente, mas ausência do aspecto vibrátil de rotura da membrana. Provavelmente, este caso teve alívio da obstrução por estiramento da membrana e consequente aumento do orifício estenótico, mecanismo também sugerido na literatura ${ }^{3,9}$.

Os casos de reestenose apresentavam características ecocardiográficas e angiográficas semelhantes às encontradas pré-dilatação e o procedimento de redilatação não implicou em dificuldades técnicas ou maior risco de complicações.

Entre os 10 pacientes que não desenvolveram reestenose, o acompanhamento ecocardiográfico mostrou redução do gradiente residual em cinco deles. O fato do gradiente médio de amostra no seguimento ser discretamente maior que o imediato pós procedimento $(36 \pm 21,5 \times 29,8 \pm 8,8-\mathrm{p}=\mathrm{NS})$ deve-se ao fato de incluirmos os casos de reestenose.

Ausência de EI deve-se, a nosso ver, à correta orientação da profilaxia e não ao grau de redução do gradiente obtido com o procedimento.

Concordantemente com a literatura ${ }^{2,3,5,9}$, não houve progressão da IAo pós-dilatação percutânea, sendo que em um caso houve redução da regurgitação valvar.

Nossa técnica difere da de outros autores ${ }^{1,3,5}$ por preferirmos utilizar sempre um balão com diâmetro semelhante ao da via de saída do VE, imediatamente abaixo de valva aórtica, ao invés de usarmos, como referência, o diâmetro do anel aórtico.

Ainda sobre a técnica, deve-se ressaltar a importância da rápida insuflação do balão para que ele se fixe ao orifício estenótico e a dilatação seja completada. A insuflação lenta impede o posicionamento adequado do balão, que é completamente expulso de cavidade ventricular esquerda pela vigorosa contração sistólica.

Nossa incidência de complicações arteriais, 2 (11,8\%) 
oclusões femorais, é baixa para procedimentos de dilatações por cateter balão. Provavelmente, isto decorre de dois fatores: a) manipulação de crianças maiores, adolescentes e jovens; b) utilização da dissecção arterial ao invés de punção e uso de introdutores de grande diâmetro.
Concluímos que, em casos corretamente selecionados, o procedimento é seguro e permite resultados satisfatórios imediatos e a médio prazo, com incidência de reestenose semelhante à encontrada com tratamento cirúrgico e que pode ser tratada com sucesso e segurança pela redilatação.

\section{Referências}

1. Arora R, Goel PK, Lochan R, Mohan JC, Khslilillsh M - Percutaneous transluminal balloon dilatation in discrete subaortic stenosis Am Heart J 1988; 116: 1091-2.

2. Wright GB, Keane JF, Nadas AS et al - Fixed subaortic stenosis in the young medical and surgical course in 83 patients. Am J Cardiol 1993; 52: 830-5.

3. Suarez de Lezo J, Pan M, Medina A et al - Immediate and follow-up results of transluminal balloon dilatation for discrete subaortic stenosis. J Am Coll Cardiol 1991; 18: 1309-15.

4. Suarez de Lezo J, Pan M, Sancho Met al - Percutaneous transluminal balloon dilatation for discrete subaortic stenosis. Am J Cardiol 1986; 58: 619-21.

5. Lababidi Z, Weinhous L, Stoeckle H, Walls JT - Transluminal balloon dilatation for discrete subaortic stenosis. Am J Cardiol 1987; 59: 423-5.

6. Maron BJ, Graham KJ, Poliac LC, NicoloffDM-Recurrence of a discrete subaortic membrane 27 years after operative resection. Am J Cardiol 1995; 76: 104-5.

7. de Vries AG, Hess J, Witsenburg M, Frohn-Mulder IME, Bogers AJJC, Bos E -
Management of fixed subaortic stenosis: A retrospective study of 57 cases. J Am Coll Cardiol 1992; 19: 1013-7.

8. Ritter SB - Discrete subaortic stenosis and balloon dilatation: The four questions revisited. J Am Coll Cardiol 1991; 18: 1316-7.

9. Feldman T, Chui YC, Carroll JD - Catheter balloon dilatation for discrete subaortic stenosis in the adult. Am J Cardiol 1987; 60: 403-5.

10. Jacob JLB, Lorga AM, Machado NCS, Coelho WMC, Garzon SAC Hemodinâmica intervencionista em cardiologia pediátrica. Arq Bras Cardiol 1991; 57(supl C): C114.

11. Jacob JLB, Machado NCS, Coelho WMC et al-Hemodinâmica intervencionista no tratamento das obstruções da via de saída de ventrículo esquerdo. Arq Bras Cardiol 1993; 61(supl II): II-58.

12. Frommelt MA, Snider AR, Bove EL, Lupinetti FM - Echocardiographic assessment of subvalvular aortic stenosis before and after operation. J Am Coll Cardiol 1992; 19: 1018-23. 